\title{
Effective Field Theory Approach to $\boldsymbol{b} \rightarrow s \ell \ell^{(\prime)}, \boldsymbol{B} \rightarrow \boldsymbol{K}^{(*)} \nu \overline{\boldsymbol{\nu}}$ and $\boldsymbol{B} \rightarrow \boldsymbol{D}^{(*)} \boldsymbol{\tau} \nu$ with Third Generation Couplings
}

\author{
Lorenzo Calibbi, ${ }^{1,2}$ Andreas Crivellin, ${ }^{3}$ and Toshihiko $\mathrm{Ota}^{4}$ \\ ${ }^{1}$ State Key Laboratory of Theoretical Physics, Institute of Theoretical Physics, \\ Chinese Academy of Sciences, Beijing 100190, People's Republic of China \\ ${ }^{2}$ Service de Physique Théorique, Université Libre de Bruxelles, C.P. 225, B-1050 Brussels, Belgium \\ ${ }^{3}$ CERN Theory Division, CH-1211 Geneva 23, Switzerland \\ ${ }^{4}$ Department of Physics, Saitama University, Shimo-Okubo 255, 338-8570 Saitama-Sakura, Japan
}

(Received 12 June 2015; published 27 October 2015)

The LHCb Collaboration reported anomalies in $B \rightarrow K^{*} \mu^{+} \mu^{-}, B_{s} \rightarrow \phi \mu^{+} \mu^{-}$, and $R(K)=B \rightarrow$ $K \mu^{+} \mu^{-} / B \rightarrow K e^{+} e^{-}$. Furthermore, BABAR, BELLE, and LHCb Collaborations found hints for the violation of lepton-flavor universality violation in $R\left(D^{(*)}\right)=B \rightarrow D^{(*)} \tau \nu / B \rightarrow D^{(*)} \ell \nu$. In this Letter we reexamine these decays and their correlations to $B \rightarrow K^{(*)} \nu \bar{\nu}$ using gauge invariant dim-6 operators. For the numerical analysis we focus on scenarios in which new physics couples, in the interaction eigenbasis, to third generation quarks and lepton only. We conclude that such a setup can explain the $b \rightarrow s \mu^{+} \mu^{-}$data simultaneously with $R\left(D^{(*)}\right)$ for small mixing angles in the lepton sector (of the order of $\pi / 16$ ) and very small mixing angles in the quark sector (smaller than $V_{c b}$ ). In these regions of parameter space, $B \rightarrow K^{(*)} \tau \mu$ and $B_{s} \rightarrow \tau \mu$ can be order $10^{-6}$. Possible UV completions are briefly discussed.

Introduction.-Thus far, the LHC Collaboration has completed the standard model (SM) of particle physics by discovering the last missing piece, the Higgs particle [1-3]. Furthermore, no significant direct evidence for physics beyond the SM has been found; i.e., no new particles were discovered. However, the LHCb Collaboration observed indirect "hints" for new physics (NP) in $B \rightarrow K^{*} \mu^{+} \mu^{-}, B_{s} \rightarrow \phi \mu^{+} \mu^{-}$, and $R(K) \equiv \operatorname{Br}\left(B \rightarrow K \mu^{+} \mu^{-}\right) /$ $\operatorname{Br}\left(B \rightarrow K e^{+} e^{-}\right)$. Furthermore, the BABAR Collaboration and also very recently the BELLE and LHCb Collaborations reported lepton-flavor universality violation in $B \rightarrow D^{(*)} \tau \nu$. These observations can be used as a guideline in the exploration of possible physics beyond the SM.

In more detail, the current experimental situation is as follows: $\mathrm{LHCb}$ reported deviations from the SM predictions [4] in $B \rightarrow K^{*} \mu^{+} \mu^{-}$[5,6] (mainly in an angular observable called $P_{5}^{\prime}$ [7]) with a significance of $2 \sigma-3 \sigma$ depending on the assumptions of hadronic uncertainties [8-11]. Also in the decay $B_{s} \rightarrow \phi \mu^{+} \mu^{-}$[12], LHCb uncovered differences compared to the SM prediction based on lattice QCD $[13,14]$ and light-cone sum rules $[15]$ of $3.1 \sigma[9,16]$. Furthermore, $\mathrm{LHCb}[18]$ found indications for the violation of leptonflavor universality, namely, $R(K)=0.745_{-0.074}^{+0.090} \pm 0.036$, in the range $1<q^{2}<6 \mathrm{GeV}^{2}$. This measurement is in tension with the theoretically clean SM prediction $R_{\mathrm{SM}}(K)=1.0003 \pm 0.0001 \quad$ [19] by $2.6 \sigma$. Combining

Published by the American Physical Society under the terms of the Creative Commons Attribution 3.0 License. Further distribution of this work must maintain attribution to the author(s) and the published article's title, journal citation, and DOI. these anomalies with all other observables for $b \rightarrow s \mu^{+} \mu^{-}$ transitions, it is found that a scenario with NP in $C_{9}^{\mu \mu}$ (corresponding to the operator $\bar{s} \gamma^{\nu} P_{L} b \bar{\mu} \gamma_{\nu} \mu$ ) but not in $C_{9}^{e e}$ is preferred compared to the SM by $4.3 \sigma$ [20].

Hints for lepton-flavor universality violating NP also comes from the BABAR Collaboration who performed an analysis of the semileptonic $B$ decays $B \rightarrow D^{(*)} \tau \nu$ [21]. Recently, these decays have also been reanalyzed by BELLE [22], and LHCb measured $B \rightarrow D^{*} \tau \nu$ [23]. Combining these measurements one finds [24] $R(D)_{\mathrm{EXP}}=0.388 \pm 0.047$, $R\left(D^{*}\right)_{\mathrm{EXP}}=0.321 \pm 0.021$. Comparing these measurements to the SM predictions [25] $R_{\mathrm{SM}}(D)=0.297 \pm 0.017$, $R_{\mathrm{SM}}\left(D^{*}\right)=0.252 \pm 0.003$, we see that there is a discrepancy of $1.8 \sigma$ for $R(D)$ and $3.3 \sigma$ for $R\left(D^{*}\right)$, and the combination corresponds approximately to a $3.8 \sigma$ deviation from the SM (compared to $3.4 \sigma$ taking into account the $B A B A R$ results only [21]).

Numerous models have been proposed in order to explain the anomalies in $b \rightarrow s \mu^{+} \mu^{-}$transitions (see, for example, Refs. [26-36] for $Z^{\prime}$ models and Refs. [37-39] for models with leptoquarks) and the deviations from the SM predictions in tauonic $B$ decays [40-49].

Alternatively, a model independent approach using higher dimensional operators has been employed, as in the model independent fits $[7,9,50]$. In this context, it has been argued that as $R(K)$ violates lepton-flavor universality, lepton flavor could also be violated in $B$ decays [51], which might be linked to neutrino oscillations $[52,53]$. While Ref. [51] considered the effect of operators at the $B$ meson scale which are invariant under electromagnetic gauge interactions only, operators invariant under the full SM gauge group [54,55] have also been considered in 
Refs. [56-62]. Here, it has been claimed than a simultaneous explanation of $R(K), R(D)$, and $R\left(D^{*}\right)$ using gauge invariant operators with left-handed fermions is possible [58,61]. For this purpose, it was assumed that in the interaction eigenbasis only couplings to the third generation exist $[51,58]$ (or are enhanced by $m_{\tau}^{2} / m_{\mu}^{2}$ compared to the second one [61]), while all other couplings are generated by the misalignment between the mass and the interaction basis (or are suppressed by small lepton-mass ratios [61]).

In this Letter we reconsider the possibility of explaining $B \rightarrow D^{(*)} \tau \nu$ and the $b \rightarrow s \mu^{+} \mu^{-}$data with higher dimensional gauge invariant operators, taking into account the constraints from $B \rightarrow K^{(*)} \nu \bar{\nu}$ and using the results of the global fit to $b \rightarrow s \mu \mu$ transitions. We extend the analysis of Ref. [61] and consider the possibility of lepton-flavor violation (LFV) while compared to Ref. [51] we include the correlations due to $S U(2)_{L}$ gauge invariance and give quantitative predictions for $B \rightarrow K^{(*)} \tau \mu$ and $B_{s} \rightarrow \tau \mu$.

Flavor observables: $b \rightarrow s \mu^{+} \mu^{-}$transitions. $-b \rightarrow s \ell_{i} \ell_{j}$ transitions are defined via the effective Hamiltonian

$$
\begin{aligned}
H_{\mathrm{eff}}^{\ell_{i} \ell_{j}} & =-\frac{4 G_{F}}{\sqrt{2}} V_{t b} V_{t s}^{*} \sum_{a=9,10}\left(C_{a}^{\ell_{i} \ell_{j}} O_{a}^{\ell_{i} \ell_{j}}+C_{a}^{\prime \ell_{i} \ell_{j}} O_{a}^{\prime \ell_{i} \ell_{j}}\right), \\
O_{9(10)}^{\ell_{i} \ell_{j}} & =\frac{\alpha}{4 \pi}\left[\bar{s} \gamma^{\mu} P_{L} b\right]\left[\bar{\ell}_{i} \gamma_{\mu}\left(\gamma^{5}\right) \ell_{j}\right],
\end{aligned}
$$

where the primed operators are obtained by exchanging $L \leftrightarrow R$.

Concerning $B \rightarrow K^{*} \mu^{+} \mu^{-}, \quad B_{s} \rightarrow \phi \mu^{+} \mu^{-}, \quad$ and $B \rightarrow$ $K \mu^{+} \mu^{-} / B \rightarrow K e^{+} e^{-}$, as already noted in Refs. [26,66], $C_{9}^{\mu \mu}<0$ and $C_{9}^{\prime \mu \mu}=0$ are preferred by the data. However, also the possibility $C_{9}^{\mu \mu}=-C_{10}^{\mu \mu}<0$ gives a good fit to data. Using the global fit of Refs. $[9,20]$ we see that at $(1 \sigma)$ $2 \sigma$ level

$$
-0.18(-0.35) \geq C_{9}^{\mu \mu}=-C_{10}^{\mu \mu} \geq(-0.71)-0.91 .
$$

Interestingly, the values of $C_{9}^{\mu \mu}, C_{10}^{\mu \mu}$ favored by $R(K)$ and $B \rightarrow K^{*} \mu^{+} \mu^{-}$lie approximately in the same range [67]. Furthermore, a good fit to the current data does not require $C_{9}^{\prime \mu \mu}$; hence, in the following we neglect operators with right-handed quark currents for simplicity.
$B \rightarrow K^{(*)} \nu \bar{\nu}$.—Following Ref. [59] we write the relevant effective Hamiltonian as

$$
\begin{aligned}
H_{\mathrm{eff}}^{\nu_{i} \nu_{j}} & =-\frac{4 G_{F}}{\sqrt{2}} V_{t b} V_{t s}^{*}\left(C_{L}^{i j} O_{L}^{i j}+C_{R}^{i j} O_{R}^{i j}\right), \\
O_{L, R}^{i j} & =\frac{\alpha}{4 \pi}\left[\bar{s} \gamma^{\mu} P_{L, R} b\right]\left[\bar{\nu}_{i} \gamma_{\mu}\left(1-\gamma^{5}\right) \nu_{j}\right],
\end{aligned}
$$

and $C_{L}^{\mathrm{SM}} \approx-1.47 / s_{w}^{2}$. In the limit of vanishing righthanded $s b$ current, the branching ratios normalized by the SM predictions read

$$
R_{K^{(*)}}^{\nu \bar{\nu}}=\frac{1}{3} \sum_{i, j=1}^{3}\left|C_{L}^{i j}\right|^{2} /\left|C_{L}^{\mathrm{SM}}\right|^{2} .
$$

The current experimental limits are $R_{K}^{\nu \bar{\nu}}<4.3[68]$ and $R_{K^{*}}^{\nu \bar{\nu}}<4.4$ [69].

$B \rightarrow D^{(*)} \tau \nu$.-The effective Hamiltonian for semileptonic $b \rightarrow c$ transitions is

$$
H_{\text {eff }}=\frac{4 G_{F}}{\sqrt{2}} V_{c b} C_{L i j}^{c b}\left[\bar{c} \gamma^{\mu} P_{L} b\right]\left[\bar{\ell}_{i} \gamma_{\mu} P_{L} \nu_{j}\right],
$$

with $C_{L i j}^{c b S \mathrm{M}}=\delta_{i j}$ (for massless neutrinos) taking into account only left-handed vector currents. In this case the ratios of branching ratios are

$$
\frac{R\left(D^{(*)}\right)_{\mathrm{EXP}}}{R\left(D^{(*)}\right)_{\mathrm{SM}}}=\sum_{j=1}^{3}\left|C_{L 3 j}^{c b}\right|^{2} / \sum_{j=1}^{3}\left|C_{L \ell j}^{c b}\right|^{2},
$$

with $\ell=e, \mu$.

Lepton-flavor violating $B$ decays.-Here we give formulas for the branching ratios of LFV $B$ decays following the analysis of Ref. [70]. We take into account only contributions from the operators $O_{9}^{(\prime) \ell \ell^{\prime}}$ and $O_{10}^{(\prime) \ell \ell^{\prime}}$ while neglecting contributions from operators with scalar currents not relevant for our analysis. For $B_{s} \rightarrow \ell^{+} \ell^{\prime-}$ (with $\ell \neq \ell^{\prime}$ ) we use the results of Ref. [71], neglecting the mass of the lighter lepton. The branching ratios for $B \rightarrow K^{(*)} \tau^{ \pm} \mu^{\mp}$, $B \rightarrow K^{(*)} \mu^{ \pm} e^{\mp}$ are computed using form factors obtained from lattice QCD in Ref. [72] (see also Refs. [14,73]). The final results read

$$
\begin{aligned}
\operatorname{Br}\left[B \rightarrow K^{(*)} \ell^{+} \ell^{\prime-}\right] & =10^{-9}\left(a_{K^{(*)} \ell \ell^{\prime}}\left|C_{9}^{\ell \ell^{\prime}}+C_{9}^{\prime \ell \ell^{\prime}}\right|^{2}+b_{K^{(*)} \ell \ell^{\prime}}\left|C_{10}^{\ell \ell^{\prime}}+C_{10}^{\prime \ell \ell^{\prime}}\right|^{2}+c_{K^{*} \ell \ell^{\prime}}\left|C_{9}^{\ell \ell^{\prime}}-C_{9}^{\prime \ell \ell^{\prime}}\right|^{2}+d_{K^{*} \ell \ell^{\prime}}\left|C_{10}^{\ell \ell^{\prime}}-C_{10}^{\prime \ell \ell^{\prime}}\right|^{2}\right), \\
\operatorname{Br}\left[B_{s} \rightarrow \ell^{+} \ell^{\prime-}\right] & =\frac{\tau_{B_{s}} m_{\ell}^{2} M_{B_{s}} f_{B_{s}}^{2}}{64 \pi^{3}} \alpha^{2} G_{F}^{2}\left|V_{t b} V_{t s}^{*}\right|^{2}\left(1-\frac{\max \left[m_{\ell}^{2}, m_{\ell^{\prime}}^{2}\right]}{M_{B_{s}}^{2}}\right)^{2}\left(\left|C_{9}^{\ell \ell^{\prime}}-C_{9}^{\prime \ell \ell^{\prime}}\right|^{2}+\left|C_{10}^{\ell \ell^{\prime}}-C_{10}^{\prime \ell \ell^{\prime}}\right|^{2}\right),
\end{aligned}
$$

TABLE I. Numerical values of the coefficients appearing in the branching ratio for $B \rightarrow K^{(*)} \ell^{+} \ell^{(I-)}$ in Eq. (8).

\begin{tabular}{lccccr}
\hline \hline$\ell \ell^{\prime}$ & $a_{K \ell \ell^{\prime}}$ & $b_{K \ell \ell^{\prime}}$ & $a_{K^{*} \ell \ell^{\prime}}$ & $b_{K^{*} \ell \ell^{\prime}}$ & $c_{K^{*} \ell \ell^{\prime}}$ \\
\hline$\tau \mu, \tau e$ & $9.6 \pm 1.0$ & $10.0 \pm 1.3$ & $3.0 \pm 0.8$ & $2.7 \pm 0.7$ & $16.4 \pm 2.1$ \\
$\mu e$ & $15.4 \pm 3.1$ & $15.7 \pm 3.1$ & $5.6 \pm 1.9$ & $5.6 \pm 1.9$ & $29.1 \pm 4.9$ \\
\hline \hline
\end{tabular}


The numerical values of the coefficients $a_{K^{(*)} \ell \ell^{\prime}}, b_{K^{(*)} \ell \ell^{\prime}}$, $c_{K^{(*)} \ell \ell^{\prime}}$ and $d_{K^{(*)} \ell \ell^{\prime}}$ are given in Table I. The formula for the branching ratio of $B_{s} \rightarrow \ell^{+} \ell^{\prime-}$ is symmetric with respect to the exchange of $C_{9}^{\left.()^{\prime}\right) \ell \ell^{\prime}} \leftrightarrow C_{10}^{(\prime) \ell \ell^{\prime}}$, while in the case of $B \rightarrow$ $K^{(*)} \ell^{+} \ell^{\prime-}$ this symmetry is broken by lepton-mass effects. There is a small difference between the theoretical prediction for the charged mode $B^{+} \rightarrow K^{(*)+} \ell^{+} \ell^{\prime-}$ and the neutral one $B^{0} \rightarrow K^{(*) 0} \ell^{+} \ell^{\prime-}$ due to the different $B$-meson lifetime $\tau_{B}$, which we neglected, fixing the numerical value of $\tau_{B}$ to the one of the neutral meson. Note that the results above are given for $\ell^{-} \ell^{\prime+}$ final states and not for the sum $\ell^{ \pm} \ell^{\prime \mp}=\ell^{-} \ell^{\prime+}+\ell^{+} \ell^{-}$to which the experimental constraints apply [74]. The only channel with $\tau \mu$ final states for which an experimental upper limit exists is

$$
\operatorname{Br}\left[B^{+} \rightarrow K^{+} \tau^{ \pm} \mu^{\mp}\right]_{\exp } \leq 4.8 \times 10^{-5} .
$$

Gauge invariant operators.-As we have previously seen, a scenario with left-handed currents only gives a good fit to data, cf. Eq. (2). In such a scenario $S U(2)_{L}$ relations are necessarily present. These relations are automatically taken into account once gauge invariant operators are considered. Therefore, let us focus on 4-fermion operators with left-handed quarks and leptons. There are two such 4-fermion operators in the effective Lagrangian,

$$
\mathcal{L}_{\text {dim }-6}=\frac{1}{\Lambda^{2}} \sum O_{X} C_{X},
$$

where $\Lambda$ is the scale of NP, which can contribute to $b \rightarrow$ sl $\ell$ transitions at tree level [54,55]:

$Q_{\ell q}^{(1)}=\left(\bar{L} \gamma^{\mu} L\right)\left(\bar{Q} \gamma_{\mu} Q\right), \quad Q_{\ell q}^{(3)}=\left(\bar{L} \gamma^{\mu} \tau_{I} L\right)\left(\bar{Q} \gamma_{\mu} \tau^{I} Q\right)$,

where $L$ is the lepton doublet and $Q$ the quark doublet, and the flavor indices are not explicitly shown here. Writing these operators in terms of their $S U(2)_{L}$ components (i.e., up-quarks, down-quarks, charged leptons, and neutrinos), we find for the terms relevant for the processes discussed in the last section (before electro-weak (EW) symmetry breaking)

$$
\begin{aligned}
\mathcal{L} \supset & \frac{C_{i j k l}^{(1)}}{\Lambda^{2}}\left(\bar{\ell}_{i} \gamma^{\mu} P_{L} \ell_{j} \bar{d}_{k} \gamma_{\mu} P_{L} d_{l}+\bar{\nu}_{i} \gamma^{\mu} P_{L} \nu_{j} \bar{d}_{k} \gamma_{\mu} P_{L} d_{l}\right) \\
& +\frac{C_{i j k l}^{(3)}}{\Lambda^{2}}\left(2 \bar{\ell}_{i} \gamma^{\mu} P_{L} \nu_{j} \bar{u}_{k} \gamma_{\mu} P_{L} d_{l}-\bar{\nu}_{i} \gamma^{\mu} P_{L} \nu_{j} \bar{d}_{k} \gamma_{\mu} P_{L} d_{l}\right. \\
& \left.+\bar{\ell}_{i} \gamma^{\mu} P_{L} \ell_{j} \bar{d}_{k} \gamma_{\mu} P_{L} d_{l}\right),
\end{aligned}
$$

where $C_{i j k l}^{(1,3)}$ are the dimensionless coefficients of the operators of Eq. (11). After EW symmetry breaking the following redefinitions of the fields are performed in order to render the mass matrices diagonal:

$d_{L} \rightarrow D^{\dagger} d_{L}, \quad u_{L} \rightarrow U^{\dagger} u_{L}, \quad \ell_{L} \rightarrow L^{\dagger} \ell_{L}, \quad \nu \rightarrow L^{\dagger} \nu$.
We define for future convenience

$$
\lambda^{(1,3)} \tilde{X}_{i j}^{(1,3)} \tilde{Y}_{k l}^{(1,3)}=L_{i^{\prime} l}^{*} L_{j^{\prime} j} D_{k^{\prime} k}^{*} D_{l^{\prime} l} C_{i^{\prime} j^{\prime} k^{\prime} l^{\prime}}^{(1,3)},
$$

where $\lambda^{(1,3)}$ are overall constants. Using constraints from the measured Cabibbo-Kobayashi-Maskawa (CKM) matrix, i.e., $V=U^{\dagger} D$, we finally obtain

$$
\begin{aligned}
C_{9}^{i j} & =-C_{10}^{i j} \\
& =\frac{\pi}{\sqrt{2} \Lambda^{2} G_{F} \alpha V_{t b} V_{t s}^{*}}\left(\lambda^{(1)} \tilde{X}_{i j}^{(1)} \tilde{Y}_{23}^{(1)}+\lambda^{(3)} \tilde{X}_{i j}^{(3)} \tilde{Y}_{23}^{(3)}\right), \\
C_{L}^{i j} & =\frac{\pi}{\sqrt{2} \Lambda^{2} G_{F} \alpha V_{t b} V_{t s}^{*}}\left(\lambda^{(1)} \tilde{X}_{i j}^{(1)} \tilde{Y}_{23}^{(1)}-\lambda^{(3)} \tilde{X}_{i j}^{(3)} \tilde{Y}_{23}^{(3)}\right), \\
C_{L i j}^{c b} & =-\frac{\lambda^{(3)}}{\sqrt{2} \Lambda^{2} G_{F}} \frac{\tilde{X}_{i j}^{(3)}}{V_{c b}} \sum_{k}\left(V_{2 k} \tilde{Y}_{k 3}^{(3)}\right)
\end{aligned}
$$

for the Wilson coefficients relevant for $b \rightarrow s \mu^{+} \mu^{-}, B \rightarrow$ $K^{(*)} \nu \bar{\nu}$ and, $B \rightarrow D^{(*)} \tau \nu$, respectively. Note that in the limit $C^{(1)}=C^{(3)}$ the contribution to $B \rightarrow K^{(*)} \nu \bar{\nu}$ vanishes.

Numerical analysis.-Since we have $C_{9}^{\tau \mu}=-C_{10}^{\tau \mu}$, we find for the LFV $B$ decays

$$
\begin{gathered}
\operatorname{Br}\left[B \rightarrow K \tau^{ \pm} \mu^{\mp}\right] / \operatorname{Br}\left[B \rightarrow K^{*} \tau^{ \pm} \mu^{\mp}\right] \approx 1, \\
\operatorname{Br}\left[B_{s} \rightarrow \tau^{ \pm} \mu^{\mp}\right] / \operatorname{Br}\left[B \rightarrow K^{*} \tau^{ \pm} \mu^{\mp}\right] \approx 0.5 .
\end{gathered}
$$

Therefore, in the following, we will just present the numerical evaluation of $\operatorname{Br}\left[B \rightarrow K^{*} \tau^{ \pm} \mu^{\mp}\right]$ while $\operatorname{Br}\left[B_{s} \rightarrow \tau^{ \pm} \mu^{\mp}\right]$ and $\operatorname{Br}\left[B \rightarrow K \tau^{ \pm} \mu^{\mp}\right]$ can be obtained by the appropriate rescaling.

We also note that $B \rightarrow K \nu \bar{\nu}$ imposes an upper limit on the absolute value of $C_{9}^{\tau \tau}=-C_{10}^{\tau \tau}$ and $C_{9}^{\tau \mu}=-C_{10}^{\tau \mu}$ valid for $C^{(3)}$ and $C^{(1)}$ separately. Neglecting the small NP contribution to $C_{L}^{\mu \mu}$ and assuming no NP in the electron channel, we find:

$$
\begin{gathered}
\frac{\left|C_{9}^{\tau \mu}\right|}{C_{L}^{\mathrm{SM}}} \leq \sqrt{4.3 \times 3 / 2} \approx 2.5, \\
\frac{\left|C_{9}^{\tau \tau}\right|}{C_{L}^{\mathrm{SM}}} \leq \sqrt{3 \times 4.3 \times 3 / 2-2}+1 \approx 5.2 .
\end{gathered}
$$

This leads to the following upper limits valid in any model generating only $C^{(3)}$ or $C^{(1)}$ :

$$
\operatorname{Br}[B \rightarrow K \tau \mu] \leq 8.3 \times 10^{-6} .
$$

However, this limit can be evaded for $C^{(3)}=C^{(1)}$. In Ref. [61] it was proposed that the minimal flavour violation (MFV)-like relation $\tilde{Y}_{22} / \tilde{Y}_{33}=m_{\tau}^{2} / m_{\mu}^{2}$ could explain $R\left(D^{(*)}\right)$ and $b \rightarrow s \mu^{+} \mu^{-}$data simultaneously. From Eq. (18) we see that this ansatz is only possible for $C^{(3)}=$ $C^{(1)}$ but not if $C^{(3)}$ or $C^{(1)}$ are separately different from zero. 
Therefore, we will focus in the following on scenarios with third generation couplings in the EW basis only, which correspond to a general rank-1 matrix in the mass eigenbasis, as suggested in Refs. $[51,58]$. In other words, we have

$$
\begin{aligned}
C_{i j k l}^{(1,3)} & =\lambda^{(1,3)} \tilde{X}_{i j} \tilde{Y}_{k l}, \\
\tilde{X} & =L^{\dagger} X L, \quad \tilde{Y}=D^{\dagger} Y D, \quad X=Y=\left(\begin{array}{lll}
0 & 0 & 0 \\
0 & 0 & 0 \\
0 & 0 & 1
\end{array}\right) .
\end{aligned}
$$

Taking into account only rotations among the second and third generation, one finds

$$
\begin{gathered}
\tilde{X}=\left(\begin{array}{ccc}
0 & 0 & 0 \\
0 & \sin ^{2}\left(\alpha_{\mu \tau}\right) & -\sin \left(\alpha_{\mu \tau}\right) \cos \left(\alpha_{\mu \tau}\right) \\
0 & -\sin \left(\alpha_{\mu \tau}\right) \cos \left(\alpha_{\mu \tau}\right) & \cos ^{2}\left(\alpha_{\mu \tau}\right)
\end{array}\right), \\
\tilde{Y}=\left(\begin{array}{ccc}
0 & 0 & 0 \\
0 & \sin ^{2}\left(\alpha_{s b}\right) & -\sin \left(\alpha_{s b}\right) \cos \left(\alpha_{s b}\right) \\
0 & -\sin \left(\alpha_{s b}\right) \cos \left(\alpha_{s b}\right) & \cos ^{2}\left(\alpha_{s b}\right)
\end{array}\right) .
\end{gathered}
$$

Note that a rotation $\sin \left(\alpha_{s b}\right) \gg V_{c b}$ would require finetuning with the up sector in order to obtain the correct CKM matrix.

$Q_{\ell q}^{(1)}$ operator.-In this case we have neutral currents only. As a consequence, there is obviously no effect in $R\left(D^{(*)}\right)$, but $b \rightarrow s \mu^{+} \mu^{-}$is directly correlated to $B \rightarrow K^{(*)} \nu \bar{\nu}$ depending on the angle $\alpha_{\mu \tau}$. Note that a change in $\alpha_{s b}$ can be compensated by a change in $\lambda^{(1)}$ and therefore does not affect the correlations among $B \rightarrow K^{(*)} \nu \bar{\nu}$ and $b \rightarrow s \mu^{+} \mu^{-}$ transitions. In Fig. 1 the regions favored by $b \rightarrow s \mu^{+} \mu^{-}$ (blue) and allowed by $B \rightarrow K \nu \bar{\nu}$ (yellow) are shown together with contour lines for $B \rightarrow K^{*} \tau \mu$ in units of $10^{-6}$. Note that $B \rightarrow K \nu \bar{\nu}$ rules out branching ratios for $B \rightarrow K^{*} \tau \mu$ above approximately $1 \times 10^{-6}$ and that the constraint from $B \rightarrow K \nu \bar{\nu}$, being inclusive in the neutrino flavors, is independent of $\alpha_{\mu \tau}$.

$Q_{\ell q}^{(3)}$ operator.-Here we have also charged currents that are related to the neutral current processes via CKM rotations. In Fig. 2 the regions allowed by $B \rightarrow K \nu \bar{\nu}$ (yellow) and giving a good fit to data for $b \rightarrow s \mu^{+} \mu^{-}$ (blue) and (at the $2 \sigma$ level) for $B \rightarrow D^{*} \tau \nu$ (red) are shown for different values of $\lambda^{(3)}$. Note that $b \rightarrow s \mu^{+} \mu^{-}$data can be explained simultaneously with $R\left(D^{(*)}\right)$ for negative $\mathcal{O}(1)$ values of $\lambda^{(3)}$ without violating the bounds from $B \rightarrow K \nu \bar{\nu}$. Again, in the regions compatible with all experimental constraints, the branching ratios of LFV $B$ decays to $\tau \mu$ final states can only be up to $\approx 10^{-6}$.

$Q_{\ell q}^{(1)}$ and $Q_{\ell q}^{(3)}$ with $\lambda^{(1)}=\lambda^{(3)}$. - In this case the phenomenology is then rather similar to the case of $C^{(3)}$ only. The major differences are that, as already mentioned, the bounds from $B \rightarrow K \nu \bar{\nu}$ are evaded and the relative contribution to $b \rightarrow s \mu \mu$ compared to $R\left(D^{(*)}\right)$ is a factor of

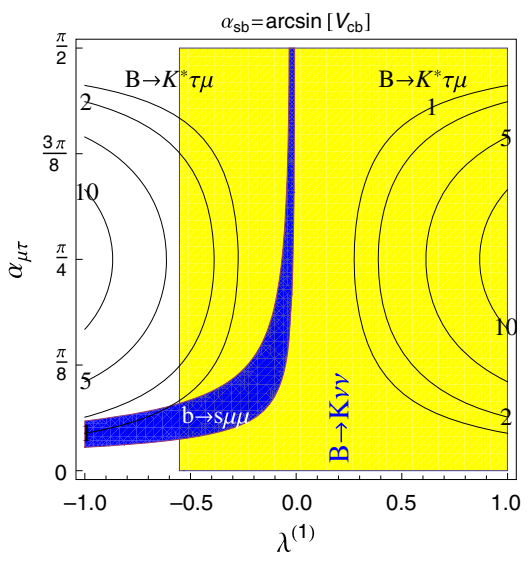

FIG. 1 (color online). Allowed regions in the $\lambda^{(1)}-\alpha_{\mu \tau}$ plane from $b \rightarrow s \mu^{+} \mu^{-}$data (blue) and $B \rightarrow K \nu \bar{\nu}$ (yellow) for $\alpha_{s b}=\arcsin \left[V_{c b}\right]$ and $\Lambda=1 \mathrm{TeV}$. Note that here changing $\alpha_{s b}$ only has the effect of an overall scaling of $\lambda^{(1)}$. The contour lines denote $\operatorname{Br}\left[B \rightarrow K^{*} \tau \mu\right]$ in units of $10^{-6}$.

2 larger. Again, $R\left(D^{(*)}\right)$ rules out very large branching ratios for lepton flavour violating $B$ decays in the regions compatible with $b \rightarrow s \mu^{+} \mu^{-}$. Note that the MFV-like ansatz [61] with additional flavour rotations phenomenologically only slightly differs from the ansatz with third generation couplings.

UV completions.-Let us briefly discuss UV completions which can give the desired coupling structure. As discussed previously, the 4-Fermi operator $Q_{\ell q}^{(3)}$ is relevant both for $R(K)$ and $R\left(D^{(*)}\right)$. If $Q_{\ell q}^{(3)}$ is mediated by a single field, then there are only four possibilities: (i) vector boson (VB) with the SM charges $\left(S U(3)_{c}, S U(2)_{L}, U(1)_{Y}\right)=(\mathbf{1}, \mathbf{3}, 0)$, (ii) scalar leptoquark (SLQ) with $(\mathbf{3}, \mathbf{3},-1 / 3)$, (iii) vector leptoquark (VLQ) with $(\mathbf{3}, \mathbf{1}, 2 / 3)$, and (iv) vector leptoquark with $(\mathbf{3}, \mathbf{3}, 2 / 3)$. The vector boson $(\mathbf{1}, \mathbf{3}, 0)$ induces only $Q_{\ell q}^{(3)}$. On the other hand, the leptoquark fields result in particular combinations of $Q_{\ell q}^{(1)}$ and $Q_{\ell q}^{(3)}$ [61]. With the assumption of the third generation coupling, the relative size of the effective couplings $\lambda^{(1,3)}$ and the signs are determined as

$\operatorname{VB}(\mathbf{1}, \mathbf{3}, 0): \lambda^{(3)}$ both positive and negative,

$$
\begin{array}{ll}
\operatorname{SLQ}(\mathbf{3}, \mathbf{3},-1 / 3): \lambda^{(1)}=3 \lambda^{(3)}, & \lambda^{(3)}>0, \\
\operatorname{VLQ}(\mathbf{3}, \mathbf{1}, 2 / 3): \lambda^{(1)}=\lambda^{(3)}, & \lambda^{(3)}<0, \\
\operatorname{VLQ}(\mathbf{3}, \mathbf{3}, 2 / 3): \lambda^{(1)}=-3 \lambda^{(3)}, & \lambda^{(3)}>0 .
\end{array}
$$

The coefficient $C_{9}^{i j}$ is proportional to $\lambda^{(1)}+\lambda^{(3)}$ and a negative value is favored by $R(K)$. Therefore, the scalar leptoquark is rejected as a candidate. To explain $R\left(D^{(*)}\right)$ simultaneously, $\lambda^{(3)}$ itself must also be negative. This condition excludes the triplet vector leptoquark. If the experimental results are explained by the operator $Q_{\ell q}^{(3)}$ 

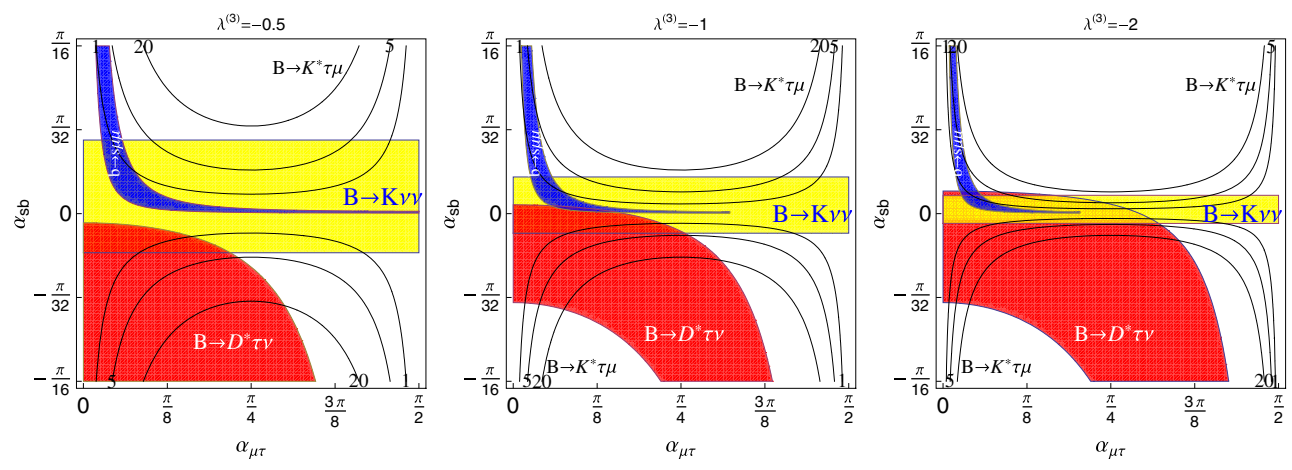

FIG. 2 (color online). Allowed regions in the $\alpha_{\mu \tau}-\alpha_{s b}$ plane from $B \rightarrow K \nu \bar{\nu}$ (yellow), $R\left(D^{*}\right)$ (red), and $b \rightarrow s \mu^{+} \mu^{-}$(blue) for $\Lambda=$ $1 \mathrm{TeV}$ and $\lambda^{(3)}=-0.5$ (left-hand panel), $\lambda^{(3)}=-1$ (middle panel) and $\lambda^{(3)}=-2$ (right-hand panel). Note that $\alpha_{s b}=\pi / 64$ roughly corresponds to the angle needed to generate $V_{c b}$, and that if $\lambda^{(3)}$ is positive, $R\left(D^{*}\right)$ and $b \rightarrow s \mu^{+} \mu^{-}$ cannot be explained simultaneously. under the assumption of third generation coupling only, the possible mediators are the triplet vector boson or the singlet vector leptoquark. According to the analysis of the previous section, a good fit to flavor data requires a mediator mass of $\mathcal{O}(1) \mathrm{TeV}$. This opens interesting prospects for the LHC, especially in the case of leptoquarks that can be produced in proton-proton collisions via color interactions and would decay to one lepton ( $\tau$ or more interestingly $\mu$ ) and one jet (possibly a $b$ jet).

Conclusions.-In this Letter we considered the effect of gauge invariant dim-6 operators with left-handed fermions on $b \rightarrow s \mu^{+} \mu^{-}, \quad B \rightarrow K^{(*)} \nu \bar{\nu}, B \rightarrow D^{(*)} \tau \nu, \quad B \rightarrow K^{(*)} \tau \mu$, and $B_{s} \rightarrow \tau \mu$. For operators with left-handed quarks and leptons, we find the correlations $\operatorname{Br}\left[B \rightarrow K \tau^{ \pm} \mu^{\mp}\right] \approx$ $\operatorname{Br}\left[B \rightarrow K^{*} \tau^{ \pm} \mu^{\mp}\right] \approx 2 \operatorname{Br}\left[B_{s} \rightarrow \tau^{ \pm} \mu^{\mp}\right]$. We showed that the anomalies in $b \rightarrow s \mu \mu$ data can be explained simultaneously with $R\left(D^{*}\right)$. For this we considered scenarios in which third generation couplings in the EW basis are present only: $\lambda^{(1)} \neq 0, \lambda^{(3)} \neq 0$, and $\lambda^{(3)}=\lambda^{(1)} \neq 0$. Taking into account $\lambda^{(1)} \neq 0$ only, $b \rightarrow s \mu^{+} \mu^{-}$data can be explained without violating bounds from $B \rightarrow K^{(*)} \nu \bar{\nu}$. However, in the allowed regions of parameter space, $\operatorname{Br}\left[B \rightarrow K^{(*)} \tau \mu\right]$ can only be up to $1 \times 10^{-6}$. In the case of $\lambda^{(3)} \neq 0, b \rightarrow s \mu^{+} \mu^{-}$data can be explained simultaneously with $R\left(D^{*}\right)$. In these regions $\operatorname{Br}\left[B \rightarrow K^{(*)} \tau \mu\right]$ can again be only up to $10^{-6}$. Finally, we considered $\lambda^{(3)}=\lambda^{(1)} \neq 0$. Such a scenario can be realized with a leptoquark in the singlet representation of $S U(2)_{L}$ (making a MFV-like ansatz for the lepton couplings possible) and constraints from $B \rightarrow K^{(*)} \nu \bar{\nu}$ are avoided. Again, LFV $B$ decays turn out to be of the same order as in the other scenarios.

A. C. and T. O. thank the ULB for hospitality during their visit in Brussels. A. C. is supported by a Marie Curie Intra-European Fellowship of the European Community's 7th Framework Programme under Contract No. PIEF-GA2012-326948. T. O. is supported by Japan Society for the Promotion of Science under KAKENHI Grant No. 26105503. L. C. thanks the Munich Institute for Astroand Particle Physics and the organizers of the workshop "Indirect Searches for New Physics in the LHC and Flavour Precision Era" for hospitality and partial financial support during the completion of this work.
Note added.-Recently, an article presenting a dynamical model with additional vector bosons and third generation couplings appeared in which $Q_{\ell q}^{(3)}$ is generated [75].

[1] G. Aad et al. (ATLAS Collaboration), Phys. Lett. B 716, 1 (2012).

[2] S. Chatrchyan et al. (CMS Collaboration), Phys. Lett. B 716, 30 (2012).

[3] We denote the SM scalar particle predicted by Brout, Englert, and Higgs as the "Higgs particle."

[4] U. Egede, T. Hurth, J. Matias, M. Ramon, and W. Reece, J. High Energy Phys. 11 (2008) 032.

[5] R. Aaij et al. (LHCb Collaboration), Phys. Rev. Lett. 111, 191801 (2013).

[6] T. L. (LHCb Collaboration), Report No. LHCb-CONF2015-002, CERN-LHCb-CONF-2015-002, 2015.

[7] S. Descotes-Genon, T. Hurth, J. Matias, and J. Virto, J. High Energy Phys. 05 (2013) 137.

[8] S. Descotes-Genon, L. Hofer, J. Matias, and J. Virto, J. High Energy Phys. 12 (2014) 125.

[9] W. Altmannshofer and D. M. Straub, Eur. Phys. J. C 75, 382 (2015).

[10] J. Lyon and R. Zwicky, arXiv:1406.0566.

[11] S. Jäger and J. Martin Camalich, arXiv:1412.3183.

[12] R. Aaij et al. (LHCb Collaboration), J. High Energy Phys. 07 (2013) 084.

[13] R. R. Horgan, Z. Liu, S. Meinel, and M. Wingate, Phys. Rev. Lett. 112, 212003 (2014).

[14] R. Horgan, Z. Liu, S. Meinel, and M. Wingate, Proc. Sci., LATTICE2014 (2015) 372.

[15] A. Bharucha, D. M. Straub, and R. Zwicky, arXiv: 1503.05534

[16] Very recently, this discrepancy increased to $3.5 \sigma$ [17].

[17] C. Linn (LHCb Collaboration), https://agenda.hepl. phys.nagoya-u.ac.jp/indico/getFile.py/access?contribId=19\& sessionId $=4 \&$ resId $=0 \&$ materialId $=$ slides \& confId $=170$.

[18] R. Aaij et al. (LHCb Collaboration), Phys. Rev. Lett. 113, 151601 (2014).

[19] C. Bobeth, G. Hiller, and G. Piranishvili, J. High Energy Phys. 12 (2007) 040.

[20] W. Altmannshofer and D. M. Straub, arXiv:1503.06199.

[21] J. Lees et al. (BABAR Collaboration), Phys. Rev. Lett. 109, 101802 (2012). 
[22] T. Chur (BELLE Collaboration), https://agenda.hepl. phys.nagoya-u.ac.jp/indico/getFile.py/access?contribId=22\& sessionId=4\&resId=0\&materialId=slides \& $\operatorname{confId}=170$.

[23] G. Ciezarek (LHCb Collaboration), https://agenda.hepl. phys.nagoya-u.ac.jp/indico/getFile.py/access?contribId=21\& sessionId=4\&resId=0\&materialId=slides\& confId $=170$.

[24] M. Rotondo, https://agenda.hepl.phys.nagoya-u.ac.jp/indico/ getFile.py/access? contribId $=9 \&$ sessionId $=19 \&$ resId $=0 \&$ materialId $=$ slides \& confId $=170$.

[25] S. Fajfer, J. F. Kamenik, and I. Nisandzic, Phys. Rev. D 85, 094025 (2012).

[26] S. Descotes-Genon, J. Matias, and J. Virto, Phys. Rev. D 88, 074002 (2013).

[27] R. Gauld, F. Goertz, and U. Haisch, Phys. Rev. D 89, 015005 (2014).

[28] A. J. Buras and J. Girrbach, J. High Energy Phys. 12 (2013) 009.

[29] R. Gauld, F. Goertz, and U. Haisch, J. High Energy Phys. 01 (2014) 069.

[30] A. J. Buras, F. De Fazio, and J. Girrbach, J. High Energy Phys. 02 (2014) 112.

[31] W. Altmannshofer, S. Gori, M. Pospelov, and I. Yavin, Phys. Rev. D 89, 095033 (2014).

[32] A. Crivellin, G. D'Ambrosio, and J. Heeck, Phys. Rev. Lett. 114, 151801 (2015).

[33] A. Crivellin, G. D’Ambrosio, and J. Heeck, Phys. Rev. D 91, 075006 (2015).

[34] C. Niehoff, P. Stangl, and D. M. Straub, Phys. Lett. B 747, 182 (2015).

[35] D. A. Sierra, F. Staub, and A. Vicente, Phys. Rev. D 92, 015001 (2015).

[36] A. Celis, J. Fuentes-Martin, M. Jung, and H. Serodio, Phys. Rev. D 92, 015007 (2015).

[37] B. Gripaios, M. Nardecchia, and S. Renner, J. High Energy Phys. 05 (2015) 006.

[38] D. Becirevic, S. Fajfer, and N. Kosnik, Phys. Rev. D 92 , 014016 (2015).

[39] I. D. M. Varzielas and G. Hiller, J. High Energy Phys. 06 (2015) 072.

[40] A. Crivellin, C. Greub, and A. Kokulu, Phys. Rev. D 86, 054014 (2012).

[41] A. Datta, M. Duraisamy, and D. Ghosh, Phys. Rev. D 86, 034027 (2012).

[42] A. Celis, M. Jung, X.-Q. Li, and A. Pich, J. High Energy Phys. 01 (2013) 054.

[43] A. Crivellin, C. Greub, and A. Kokulu, Phys. Rev. D 87, 094031 (2013).

[44] X.-Q. Li, Y.-D. Yang, and X.-B. Yuan, Phys. Rev. D 89, 054024 (2014).

[45] G. Faisel, Phys. Lett. B 731, 279 (2014).

[46] M. Atoui, V. Mornas, D. Beirevic, and F. Sanfilippo, Eur. Phys. J. C 74, 2861 (2014).

[47] Y. Sakaki, R. Watanabe, M. Tanaka, and A. Tayduganov, Phys. Rev. D 88, 094012 (2013).

[48] I. Dorner, S. Fajfer, N. Konik, and I. Niandic, J. High Energy Phys. 11 (2013) 084.

[49] P. Biancofiore, P. Colangelo, and F. De Fazio, Phys. Rev. D 89, 095018 (2014).
[50] T. Hurth, F. Mahmoudi, and S. Neshatpour, J. High Energy Phys. 12 (2014) 053.

[51] S. L. Glashow, D. Guadagnoli, and K. Lane, Phys. Rev. Lett. 114, 091801 (2015).

[52] S. M. Boucenna, J. W. F. Valle, and A. Vicente, arXiv: 1503.07099.

[53] Lepton flavor violating $B$ decays in leptoquark models have been studied in Ref. [39] and in $Z^{\prime}$ models in Ref. [70].

[54] W. Buchmuller and D. Wyler, Nucl. Phys. B268, 621 (1986).

[55] B. Grzadkowski, M. Iskrzynski, M. Misiak, and J. Rosiek, J. High Energy Phys. 10 (2010) 085.

[56] S. Fajfer, J. F. Kamenik, I. Nisandzic, and J. Zupan, Phys. Rev. Lett. 109, 161801 (2012).

[57] R. Alonso, B. Grinstein, and J. Martin Camalich, Phys. Rev. Lett. 113, 241802 (2014).

[58] B. Bhattacharya, A. Datta, D. London, and S. Shivashankara, Phys. Lett. B 742, 370 (2015).

[59] A. J. Buras, J. Girrbach-Noe, C. Niehoff, and D. M. Straub, J. High Energy Phys. 02 (2015) 184.

[60] C.-J. Lee and J. Tandean, J. High Energy Phys. 08 (2015) 123.

[61] R. Alonso, B. Grinstein, and J. M. Camalich, arXiv: 1505.05164

[62] For an analogous analysis in the lepton sector, see Refs. [63-65].

[63] A. Crivellin, S. Najjari, and J. Rosiek, J. High Energy Phys. 04 (2014) 167.

[64] A. Crivellin, M. Hoferichter, and M. Procura, Phys. Rev. D 89, 093024 (2014).

[65] G. M. Pruna and A. Signer, J. High Energy Phys. 10 (2014) 14.

[66] S. Descotes-Genon, J. Matias, and J. Virto, Proc. Sci., EPSHEP2013 (2013) 361.

[67] Note that the fit to Eq. (2) includes muon data only. However, as $B \rightarrow K e^{+} e^{-}$agrees rather well with the SM prediction, the effect on the global fit is expected to be small. Also, the latest LHCb result for $B_{s} \rightarrow \phi \mu^{+} \mu^{-}$[17], which would slightly increase the tension with the SM, is not included in the fit.

[68] J. Lees et al. (BABAR Collaboration), Phys. Rev. D 87, 112005 (2013).

[69] O. Lutz et al. (Belle Collaboration), Phys. Rev. D 87, 111103 (2013).

[70] A. Crivellin, L. Hofer, J. Matias, U. Nierste, S. Pokorski, and J. Rosiek, Phys. Rev. D 92, 054013 (2015).

[71] A. Dedes, J. Rosiek, and P. Tanedo, Phys. Rev. D 79, 055006 (2009).

[72] C. Bouchard, G. P. Lepage, C. Monahan, H. Na, and J. Shigemitsu (HPQCD Collaboration), Phys. Rev. D 88, 054509 (2013).

[73] R. R. Horgan, Z. Liu, S. Meinel, and M. Wingate, Phys. Rev. D 89, 094501 (2014).

[74] Y. Amhis et al. (Heavy Flavor Averaging Group), arXiv: 1412.7515.

[75] A. Greljo, G. Isidori, and D. Marzocca, J. High Energy Phys. 07 (2015) 142. 